\title{
A Novel Chebyshev Series Fed Linear Array with High Gain and Low Sidelobe Level for WLAN Outdoor Systems
}

\author{
Tang The Toan ${ }^{1}$, Nguyen Minh Tran ${ }^{2}$, Truong Vu Bang Giang ${ }^{3}$ \\ ${ }^{1}$ Faculty of Electronic Engineering, Hanoi University of Industry, Hanoi, Vietnam \\ 2 Sungkyunkwan University, Suwon, Korea \\ 3 Vietnam National University, Hanoi, Vietnam
}

Correspondence: Truong Vu Bang Giang, giangtvb@vnu.edu.vn

Communication: received 8 November 2017, revised 18 March 2018, accepted 19 March 2018

Online publication: June 20 2018, Digital Object Identifier: 10.21553/rev-jec.187

The associate editor coordinating the review of this article and recommending it for publication was Dr. Vu Van Yem.

\begin{abstract}
This paper proposes a novel high gain and low sidelobe level (SLL) linear microstrip array antenna for outdoor WLAN applications. The antenna consists of two main parts, which are a linear array and a reflector. The linear array comprises of 10 elements, those have been designed on Rogers RT/Duroid 5870tm with the dimensions of $422 \times 100 \times$ $10.15 \mathrm{~mm}^{3}$. To gain low SLLs, a series fed network was designed to have the output signals being proportional to the Chebyshev distributions (with preset SLL of $-30 \mathrm{~dB}$ ). Furthermore, Yagi antenna theory has been applied by adding directors above every single element to increase the directivity of the single element. The reflector has been constructed at the back of the proposed structure. Simulation results show that the array can provide high gain of $17.5 \mathrm{dBi}$ and a low SLL of $-26 \mathrm{~dB}$. A prototype has been fabricated and measured. Good agreements between simulation and measurement data have been obtained.
\end{abstract}

Keywords- Fan-beam, linear array, low sidelobe.

\section{Introduction}

Nowadays, due to the increasing requirements of users in throughput, data rate and robustness, recent $\mathrm{Wi}-\mathrm{Fi}$ standards such as IEEE $802.11 \mathrm{n} /$ ac have been moved to the $5 \mathrm{GHz}$ spectrum instead of $2.4 \mathrm{GHz}$ as in the previous ones. Although it allows wider bandwidth and higher data rate, the use of $5 \mathrm{GHz}$ has to face the difficulty of propagation loss. Actually, the path loss of this band is approximately $8 \mathrm{~dB}$ higher than that of 2.4 GHz band. Therefore, for outdoor applications, the antennas are often required to attain more than $12 \mathrm{dBi}$ as in the commercial products. Additionally, it is also noticed that the beamwidth of the antenna is usually inversely proportional to the gain. Hence, the fan-beam antenna can be a good choice to alleviate this problem.

Printed antenna arrays are usually used in telecommunication systems such as point to point and point to multipoint, in radar microwave, and millimeter systems. However, combining antenna elements in an array may lead to larger in size and the SLL will be high, which is the main drawback of such kind of antenna. The high SLL in the array may be caused by: tolerances in fabrication, mutual coupling between radiating elements, limitations in feasibility of feeding network realization, surface wave effect as well as parasitic radiation from a feeding network. Several techniques to reduce the SLL of the array have been investigated and proposed in the literature. In the digital beamforming (smart antennas) or radar systems, Binomial, Cheby- shev and Taylor distributions have been commonly applied in power excitation of the array to get low SLL. In the ordinary arrays, the same thing can be achieved by designing the feeding network which has output power corresponding to these distributions. Among several types of array feeding structures, there are two common ones: corporate and series fed. The discontinuities, bends and power dividers in the corporate fed array may cause spurious radiation that raises the SLL to high levels, especially in large arrays. In opposite with the corporate one, the series fed, which employs shorter and fewer transmission lines, leads to an array antenna with smaller size, lower attenuation loss and spurious radiation from the feed lines.

Recently, several attempts have been done to suppress the SLL in the printed antenna arrays [1-10]. A novel aperture coupled microstrip antenna array has been proposed in [1]. The antenna consists of a total 100 microstrip elements that are arranged in two rows of 50 elements, with element spacing of 0.51 free-space wavelength. A SLL of $-20.9 \mathrm{~dB}$ has been obtained. However, because of using a large number of elements, the size of the whole array is considerably large while the SLL is not low. In [2], Y. P. Saputra, et al., have introduced a sidelobe suppression method for X-band antenna array by designing a novel feeding network with Chebyshev distribution. The array operates at $9.3 \mathrm{GHz}$ and can give $-19.4 \mathrm{~dB}$ SLL. As the complexity of the feeding network and the limited number of elements, the gain is only $12.3 \mathrm{dBi}$ and the SLL is still high. Another 
6 elements antenna array with linear series fed has been presented in [3]. A wideband of $6.5 \%$ has been obtained, but the SLL and the gain acquired are still not good, which are $-20 \mathrm{~dB}$ and $14.2 \mathrm{dBi}$, respectively. Lower SLLs have been tackled and obtained in [4, 5]. By using the optimized distribution coefficients through differential evolution algorithm, a wideband and lower SLL have been achieved in slot antenna with series fed network in [4]. The antenna includes of 10 elements which are arranged in a series feeding network. The SLL and half power beam-width (HPBW) are $-25.3 \mathrm{~dB}$ and $8.4^{\circ}$, respectively. However, the gain achieved is only $14.5 \mathrm{dBi}$. In addition, the authors in [5] have proposed a low SLL and wideband series fed dielectric resonator antenna (DRA) array. The proposal can get a very low SLL of $-30 \mathrm{~dB}$ and high gain of $19 \mathrm{dBi}$. Nevertheless, the large number of elements (up to 22 dielectric resonators have been used) result in a large size. Besides, the fabrication could be complicated as complex design techniques have been used. Similarly, two other DRA arrays have been introduced in [6, 7]. The array in [6] works at $60 \mathrm{GHz}$, while the one in [7] operates at 7.4 GHz. The SLL are $-27.7 \mathrm{~dB}$ and $-23 \mathrm{~dB}$, respectively. R. Bayderkhani and $H$. R. Hassani have presented two linear series fed Yagi-like array samples in $[8,9]$. The arrays have 22 similar Yagi line elements, which can provide the gain of $15.3 \mathrm{dBi}$ and the SLL of about $-27 \mathrm{~dB}$. In spite of using a huge number of single elements and the Chebyshev distribution with the SLL preset to $-35 \mathrm{~dB}$, the gain and the SLL is not noticeably good. Hassani and his other colleagues have continuously proposed two other low SLL arrays [10,11]. The sample in [10] has the SLL of $20 \mathrm{~dB}$, while the lower SLL $(-33.2 \mathrm{~dB})$ has been obtained in [11]. However, the gain in [10] is remarkably high with $18.2 \mathrm{~dB}$ compared to just $15.9 \mathrm{dBi}$ in [11].

In this paper, a high gain, low SLL, microstrip linear antenna array will be introduced. The design procedure from single element, feeding network and the complete array will be specifically demonstrated. The array consists of 10 single elements, which are double-sided printed dipoles (DSPD). The low SLL is obtained by designing the series fed network with the output power corresponding to the Chebyshev coefficients (preset SLL of $-30 \mathrm{~dB}$ ). As can be seen from the simulation results, the antenna operates well at $5.5 \mathrm{GHz}$ with the bandwidth of $212 \mathrm{MHz}$. Moreover, a high gain of $17.5 \mathrm{dBi}$ has also been acquired, while the SLL is low at $-26 \mathrm{~dB}$. Fabrication and measurement have been done, and simulated results have been validated with the corresponding measured data. Good agreements between simulation and measurement have been shown.

\section{Antenna Design and Structure}

\subsection{Design of the Single Element}

To build an array, a single element should be first selected and designed. In this work, to guarantee the requirements in both size and performance, DSPD has been used as the single element of the array. In

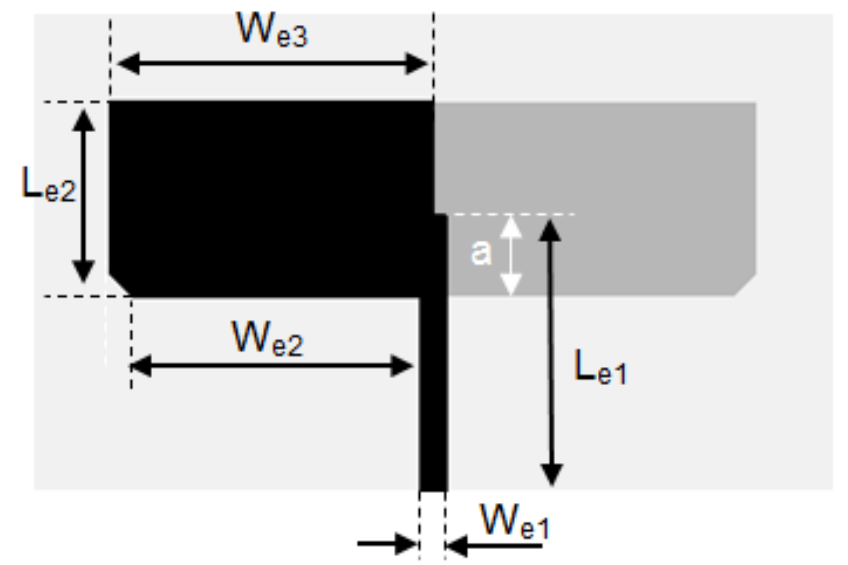

Figure 1. The proposed single element (black: top, grey: bottom).

paper [12], the authors have presented and analyzed this kind of antenna in details. The fundamental DSDP consists of a parallel strip line and two arms, which are printed on the opposite surface of the dielectric sheet. Figure 1 gives the proposed DSDP used as single elements in the array construction. The quarter wavelength transform, a prevalent matching technique, is applied in this work. Indeed, the strip line with the length of a quarter wavelength serves as a matching part between the patch and the input impedance. Therefore, the impedance (the width) and the length of the strip line can be obtained. The length of the feed line and the patch can be given by approximately a quarter of the wavelength $\left(\lambda_{g} / 4=c / 4 f_{0} \sqrt{\epsilon_{r}}\right)$. The width of the patch can be calculated by:

$$
Z_{a} \approx 90 \frac{\epsilon_{r}^{2}}{\epsilon_{r}-1}\left(\frac{L}{W}\right)^{2}
$$

where $Z_{a}=Z_{0}^{2} / Z_{i n}, Z_{i n}=50 \Omega, Z_{0 \text { (parallelstrip) }}=$ $2 Z_{0 \text { (microstrip) }}$ with $h=d / 2$.

In this paper, the DSDP is placed on the Roger RTDuroid 5870tm (thickness of $1.575 \mathrm{~mm}$ and $\epsilon_{r}=$ 2.33), and has been adjusted to work at $5.5 \mathrm{GHz}$ with the impedance $Z_{d}=100 \Omega$ at the center frequency. The final dimensions of the single element are given in Table I.

Table I

The Parameters of the Single Element

\begin{tabular}{|c|c|c|c|}
\hline Parameters & Value $\mathbf{( m m )}$ & Paremeters & Value $(\mathbf{m m})$ \\
\hline$W_{e 1}$ & 0.8 & $L_{e 1}$ & 8.5 \\
\hline$W_{e 2}$ & 9.1 & $L_{e 2}$ & 6.0 \\
\hline$W_{e 3}$ & 10.0 & $\mathrm{a}$ & 2.5 \\
\hline
\end{tabular}

The simulated $S_{11}$ and radiation pattern of the DSDP are shown in Figure 2 and Figure 3, respectively. It is clear that the DSDP can work well at $5.5 \mathrm{GHz}$ with the gain of $5.27 \mathrm{dBi}$. This DSDP will be used as the single element of the array in this work.

\subsection{Design of the Feeding Network}

This section will present the procedure to design the feeding network using in the array. The series fed 


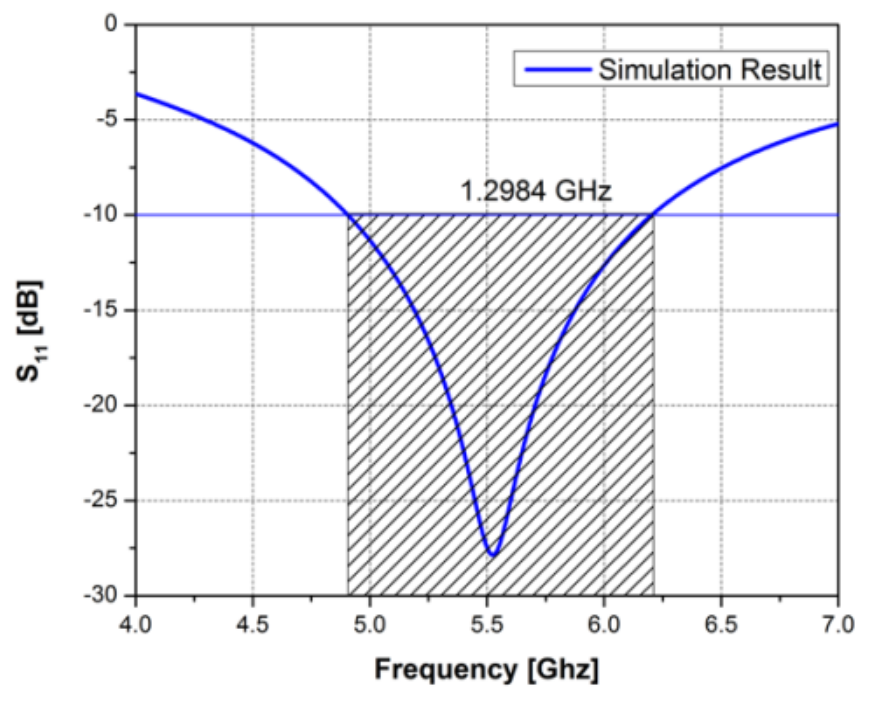

Figure 2. Simulated $S_{11}$ of the DSPD.

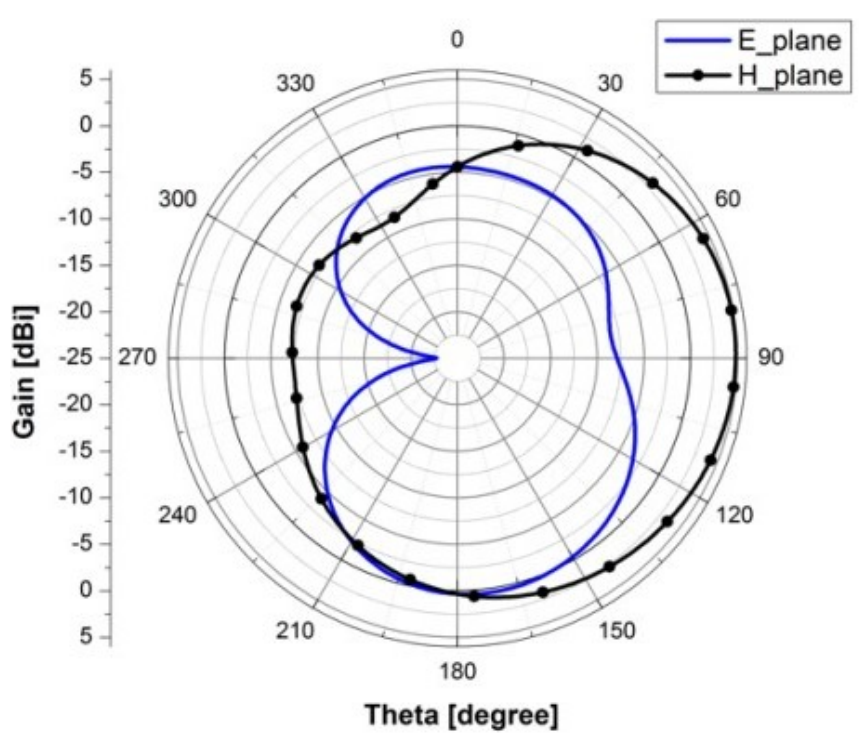

Figure 3. Radiation pattern of the DSPD.

network for the linear array is designed to match with the single element in the previous section to form a $10 \times 1$ array antenna. The Chebyshev distribution with preset SLL of $-30 \mathrm{~dB}$ has been utilized as the output coefficients' target. In order to obtain the Chebyshev distribution in the series fed network, different shunt stubs have been added to the feed line so that the amount of signal flowing out each output port can be easily controlled. The operation and the equivalent circuit of the stub in the antenna are given in Figure 4. According to [13], the equivalent shunt capacitor of shunt stub can be calculated by:

$$
Y_{i n}=j Y_{c} \tan \left(\frac{2 \pi}{\lambda_{g}} l\right) \approx j Y_{c}\left(\frac{2 \pi}{\lambda_{g}} l\right)=j \omega\left(\frac{Y_{c} l}{v_{p}}\right),
$$

where $Y_{c}$ is the equivalent admittance of the stub, $l$ is the length of the stub, $v_{p}$ is the phase velocity, $\lambda_{g}$ is the wavelength in the substrate.

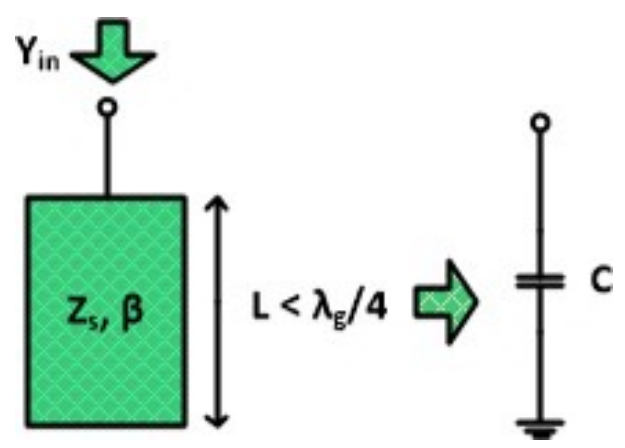

Figure 4. The equivalent capacitor of shunt stub.

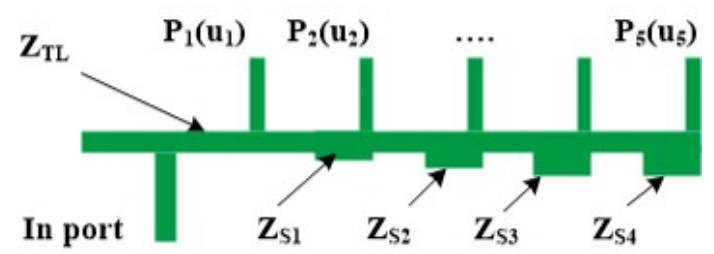

(a) The feed model with shunt stubs.

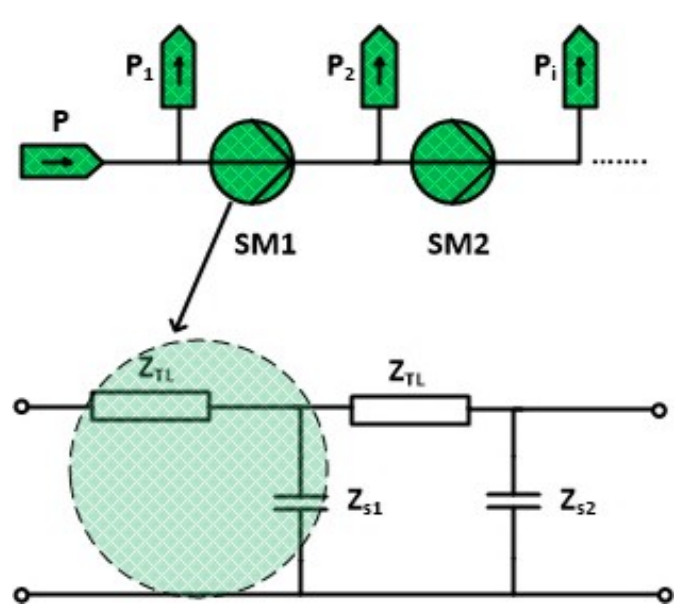

(b) The equivalent circuit.

Figure 5. The series fed network and its equivalent circuit.

As the symmetrical properties of Chebyshev distribution, the proposed feeding network has a mirror like structure. Indeed, the feed has a main line which is fed by the $50 \Omega$ line at the center. To form the symmetrical geometry, the same feeding line and shunt stubs are equally distributed on each side of the central line. These stubs serve as shunt capacitors and play as impedance matching to control the output amplitude excited at each element. Due to the effect of shunt stubs in the impedance matching point, the $S$ parameters at each port related to the input port will be easily handled. It means that the energy flowing out each output port can be controlled [14]. The feed model at one side and the equivalent circuit are shown Figure 5.

In this work, the feeding network has been designed for $10 \times 1$ antenna array with the Chebyshev amplitudes corresponding to SLL of $-30 \mathrm{~dB}$. The Chebyshev amplitudes for 10 elements are given in the Table II. 
Table II

Chebyshev Amplitude Weights for $10 \times 1$ Linear ArRay $(\mathrm{SLL}=$ $-30 d B)$

\begin{tabular}{|l|c|c|c|c|c|}
\hline Element No. & $u_{1}$ & $u_{2}$ & $u_{3}$ & $u_{4}$ & $u_{5}$ \\
\hline Amplitude & 1 & 0.8780 & 0.6692 & 0.43 & 0.2575 \\
\hline $\begin{array}{c}\text { Characteristic } \\
\text { impedances }\end{array}$ & $Z_{T L}$ & $Z_{s 1}$ & $Z_{s 2}$ & $Z_{s 3}$ & $Z_{s 4}$ \\
\hline Element No. & $u_{6}$ & $u_{7}$ & $u_{8}$ & $u_{9}$ & $u_{10}$ \\
\hline Amplitude & 1 & 0.8780 & 0.6692 & 0.43 & 0.2575 \\
\hline $\begin{array}{c}\text { Characteristic } \\
\text { impedances }\end{array}$ & $Z_{T L}$ & $Z_{s 5}$ & $Z_{s 6}$ & $Z_{s 7}$ & $Z_{s 8}$ \\
\hline
\end{tabular}

Table III

Characteristic Impedances of the Series Fed Network

\begin{tabular}{|l|c|c|c|c|c|}
\hline $\begin{array}{c}\text { Characteristic } \\
\text { impedances }\end{array}$ & $Z_{T L}$ & $Z_{s 1}$ & $Z_{s 2}$ & $Z_{s 3}$ & $Z_{s 4}$ \\
\hline Value $(\Omega)$ & 178 & 151.11 & 118.98 & 76.454 & 45.78 \\
\hline $\begin{array}{c}\text { Characteristic } \\
\text { impedances }\end{array}$ & $Z_{T L}$ & $Z_{s 5}$ & $Z_{s 6}$ & $Z_{s 7}$ & $Z_{s 8}$ \\
\hline Value $(\Omega)$ & 178 & 151.11 & 118.98 & 76.454 & 45.78 \\
\hline
\end{tabular}

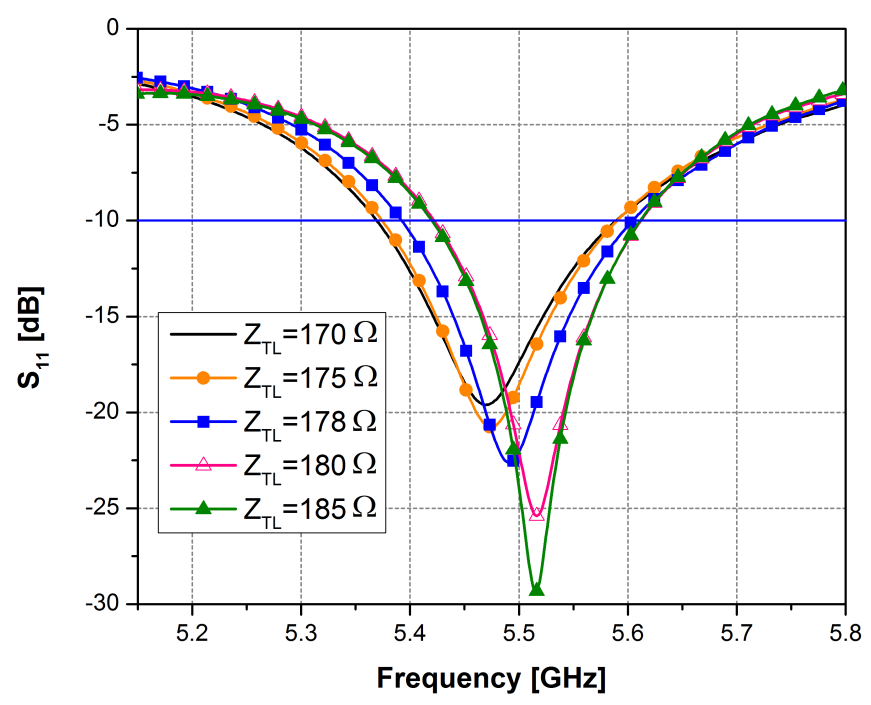

Figure 6. The simulated return loss of different main line impedances.

The impedance of the shunt stubs $\left(Z_{s 1}\right.$ to $\left.Z_{s 4}\right)$ can be determined using theory given in [7] as follow: $Z_{s 1} / Z_{T L}=u_{2} / u_{1}=0.8780 ; Z_{s 2} / Z_{T L}=u_{3} / u_{1}=0.6692 ;$ $Z_{s 3} / Z_{T L}=u_{4} / u_{1}=0.4300 ; Z_{s 4} / Z_{T L}=u_{5} / u_{1}=0.2575$, in which $u_{n}$ is the output current corresponding to the $n^{\text {th }}$ element. For determining the value of impedances and the value of shunt stubs, the main line should be firstly selected. The different values of the main line have been simulated, and the results are given in Figure 6 . The wider bandwidth has been achieved with the main line impedance of $178 \Omega$. Hence, the main line has been designed with the impedance of $178 \Omega$. Eventually, these values of $Z_{s i}$ have been calculated as given in Table III.

Figure 7 shows the final series fed network of 10 elements designed on Roger RT/Duroid 5870tm substrate (the height of $1.575 \mathrm{~mm}$ and the permeability of 2.33) with the dimensions of $55 \times 385 \times 1.575 \mathrm{~mm}^{3}$.
Table IV

Parameters of $10 \times 1$ Series Fed Network

\begin{tabular}{|c|c|c|c|}
\hline Parameters & Value (mm) & Parameters & Value (mm) \\
\hline $\mathrm{d}$ & 38.8 & $W_{s 4}$ & 1.14 \\
\hline$l_{s}$ & 3.75 & $W_{s 5}$ & 0.80 \\
\hline$W_{s 1}$ & 7.00 & $W_{1}$ & 2.00 \\
\hline$W_{s 2}$ & 5.13 & $W_{2}$ & 0.80 \\
\hline$W_{s 3}$ & 3.30 & $g$ & 7.00 \\
\hline
\end{tabular}

Table $\mathrm{V}$

The Simulated Amplitudes and Phases of the Series Fed NETWORK

\begin{tabular}{|l|c|c|c|c|c|}
\hline Element No. & $u_{1-6}$ & $u_{2-7}$ & $u_{3-8}$ & $u_{4-9}$ & $u_{5-10}$ \\
\hline Amplitude & 1.000 & 0.9238 & 0.6726 & 0.4150 & 0.1863 \\
\hline Phase $\left(^{\circ}\right)$ & -50.48 & -53.67 & -53.10 & -55.75 & -51.12 \\
\hline
\end{tabular}

Parameters of $10 \times 1$ series fed network are given in Table IV [15].

Table $\mathrm{V}$ gives the simulated amplitude and phase data at each output port. It is clear that the simulated amplitude coefficients approached to the Chebyshev distribution with negligible differences. The normalized radiation pattern of simulated coefficients from the feed and the corresponding one from theory have been presented in Figure 8. This series fed network can be combined with single elements to construct a linear antenna array which has the SLL suppressed to $-27 \mathrm{~dB}$.

\subsection{Design of the Array}

The array structure is obtained by combining optimized single elements and the series fed network developed in the previous sections.

So as to increase the gain, two techniques are being applied in the design of the array. Firstly, as the inspiration of Yagi theory, directors are being added into the elements of the array [16]. Hence, according to the theory given in [16] that the directors in Yagi antenna should be around $0.4 \lambda$ to $0.48 \lambda$, each single element has been integrated with three directors, which have the same size of $2.5 \times 15 \mathrm{~mm}\left(0.42 \lambda_{g} \times 0.07 \lambda_{g}\right)$, at both sides of the DSDP. The number of directors can be designed larger subject to the trade off between gain and dimensions required in the array. The simulated results of the gain and the SLL with respect to the sample with and without directors will be specifically presented and discussed. Secondly, a reflector is being placed at the back of the main array with the distance of $\lambda_{g} / 4(7.2 \mathrm{~mm})$ so as to boost up the gain of the whole array. The overall size of the proposed array is $422 \times 100 \times 10.15 \mathrm{~mm}^{3}$ as given in Figure 9 .

The optimized array has been fabricated and measured in the laboratory as shown in the Figure 10. In Section 3, the detailed results will be compared and discussed. 


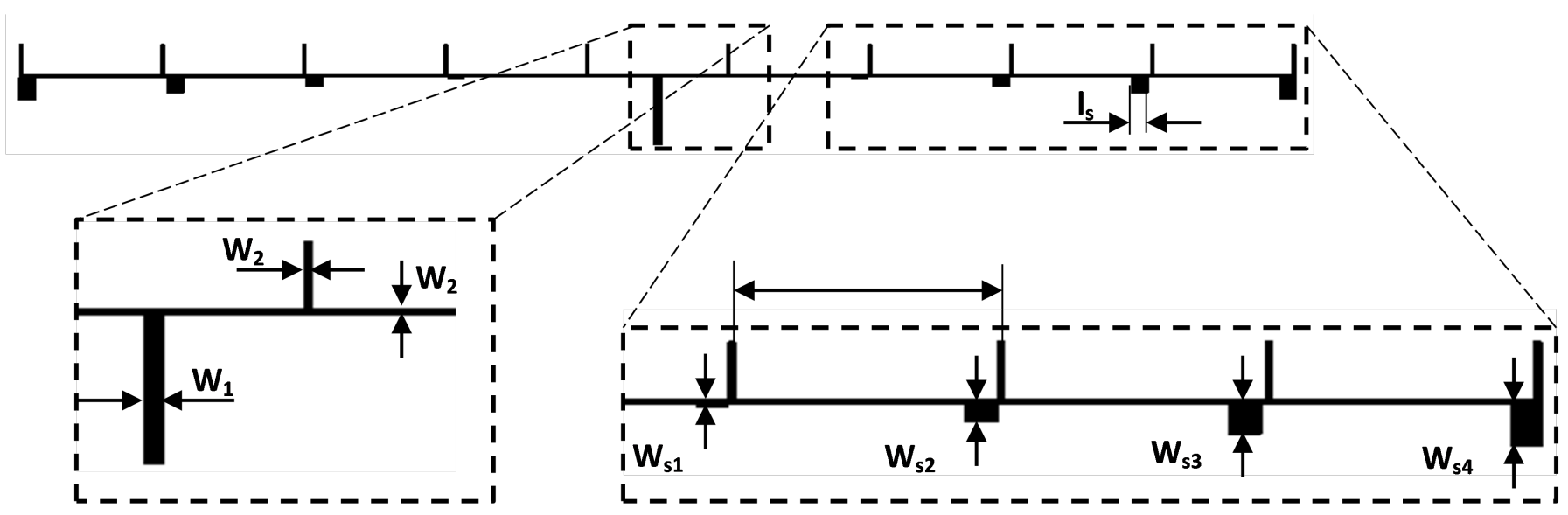

Figure 7. $10 \times 1$ series fed network.

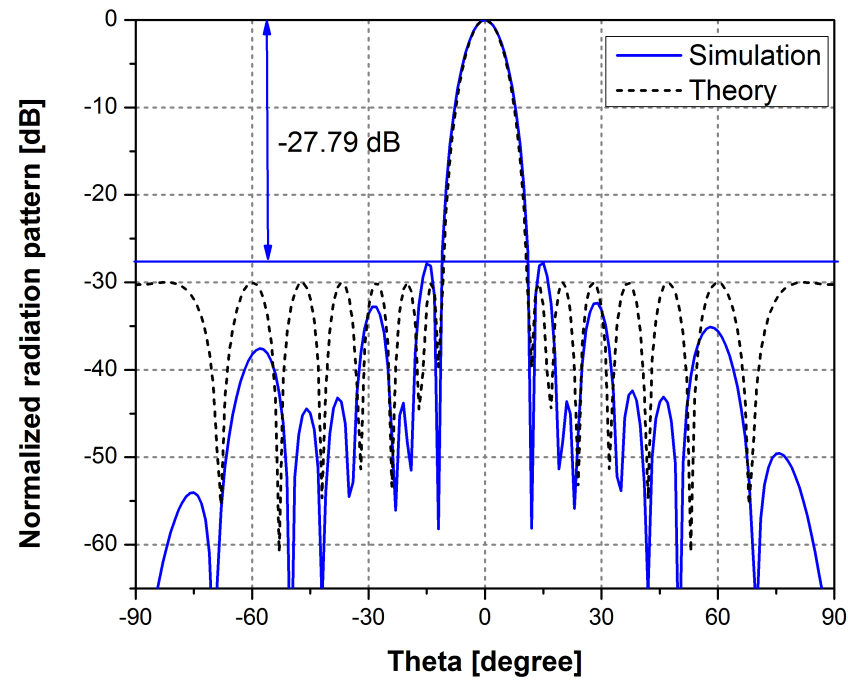

Figure 8. Normalized radiation patterns with respect to the output power coefficient (solid line) and theoretical one (dotted line).

\section{Results ANd Discussions}

\subsection{Simulation Results}

Figure 11 shows the simulated $S_{11}$ of the array. The proposed antenna operates at $5.5 \mathrm{GHz}$ with the bandwidth of $212 \mathrm{MHz}$ at $-10 \mathrm{~dB}$ of $S_{11}$.

The radiation pattern, gain and SLL over the operating frequency have also been demonstrated in Figures 12 and 13. As can be seen from Figure 12, a narrow $\mathrm{HPBW}$ of $10.40^{\circ}$ is acquired over $5.4 \mathrm{GHz}-5.65 \mathrm{GHz}$ band.

The stable gain at about $17 \mathrm{dBi}$, and the SLL, which is lower than $-20 \mathrm{~dB}$, are obtained over the bandwidth as given in Figure 13. At $5.5 \mathrm{GHz}$, the gain of $17.5 \mathrm{dBi}$ and low SLL of $-26.0 \mathrm{~dB}$ have been acquired. In comparison with those from theory and the feeding in [15], the SLL of the array in this paper is $1.79 \mathrm{~dB}$ higher (see Figure 8). It proves the capability of applying the series fed network to simultaneously obtain high gain and low SLL arrays.

It is noticable that the SLL and gain in the case with added directors are better compared to that of the array without directors. Therefore, the benefits of high gain

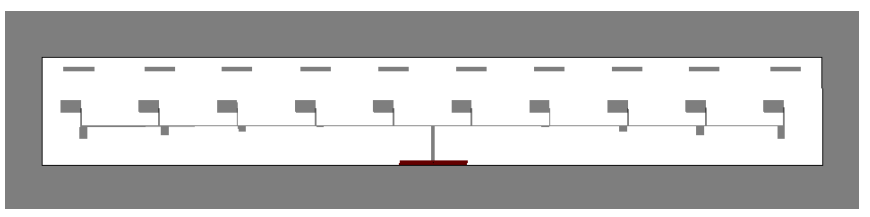

(a) Top view.

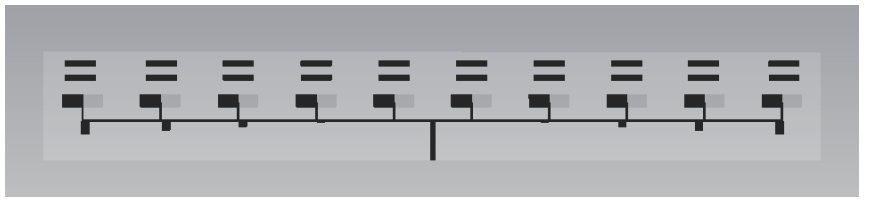

(b) Bottom view.

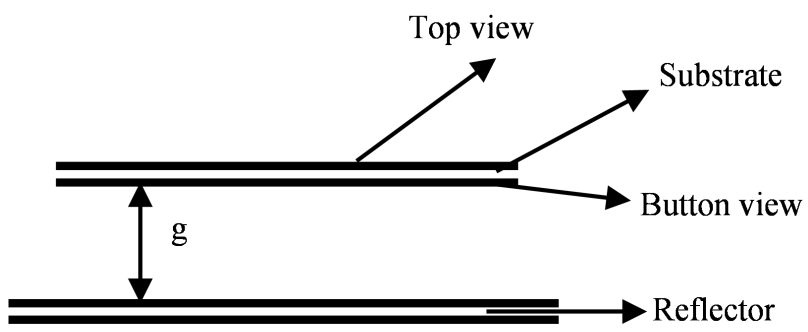

(c) Side view.

Figure 9. Structure of the proposed array.

in Yagi antenna has been successfully leveraged and employed in this proposal, and approximate $1 \mathrm{~dB}$ gain higher is achieved by using 3 additional directors. The gain over operating frequency band of the sample with and without the reflector is shown in Figure 14. The array with the reflector definitely has higher gain (about $3 \mathrm{~dB}$ ) compared to the one having no reflector. Hence, the use of the reflector has a great advantage in terms of gain enhancing. Furthermore, it is also demonstrated that the array radiates with high efficiency (almost more than $90 \%$ ) in the operating range.

The effect of the reflector has also been investigated and analyzed as shown in Figure 15. The graph demonstrates that the gain and SLL vary with respect to the different distances between the reflector and the array. It is clear that the gain rises slowly before reaching the peak of $17.5 \mathrm{dBi}$ at a quarter wavelength distance. Then, it gradually falls down to around $16 \mathrm{dBi}$ with 


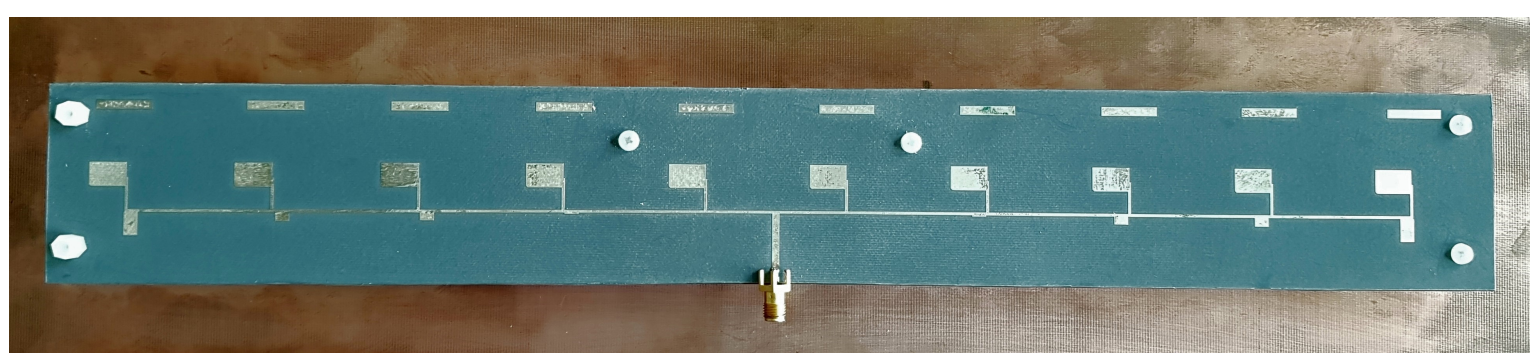

Figure 10. The fabricated antenna array.

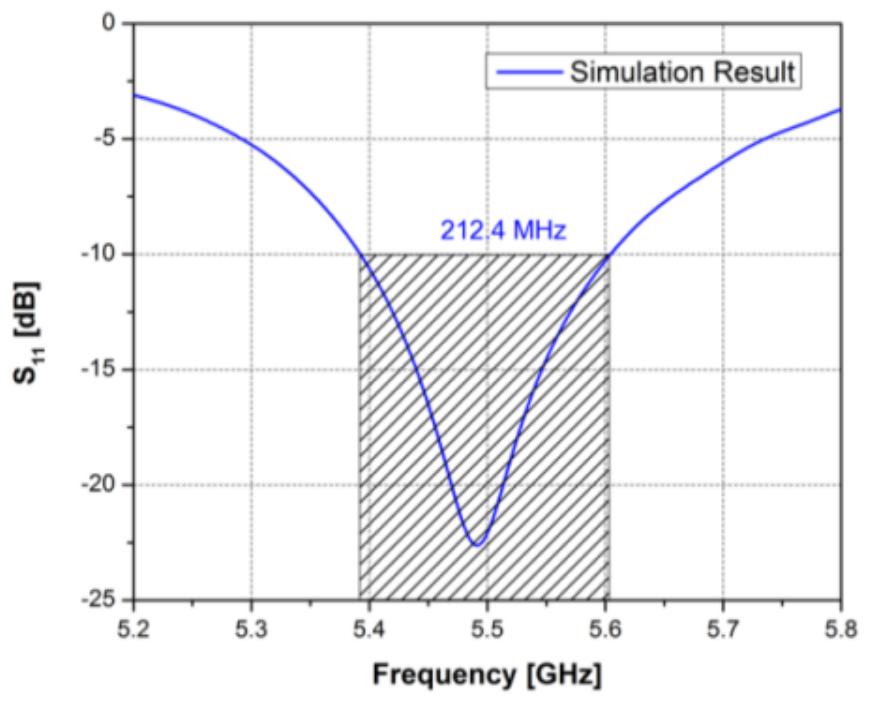

Figure 11. Simulated $S_{11}$ of the array.

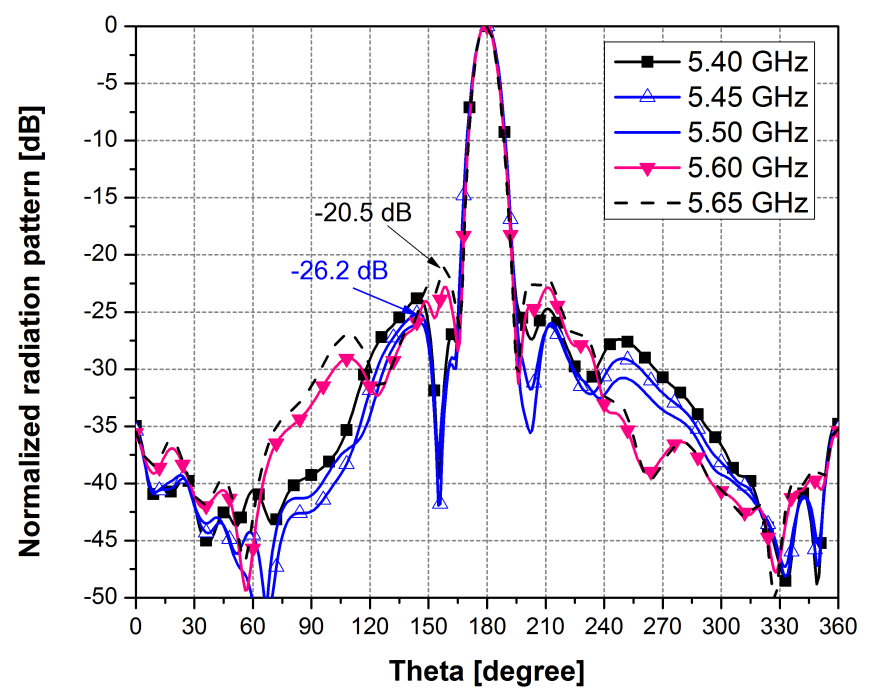

Figure 12. The radiation pattern over different frequencies.

one wavelength distance. Similarly, the SLL also drops to the bottom at around $-26.5 \mathrm{~dB}$ around the distance of a quarter wavelength. Hence, the optimized distance between the reflector and the array is transparently a quarter wavelength.

\subsection{Experimental Results}

The measurement of the prototype has been done and the measured data has been compared to that of the simulation.

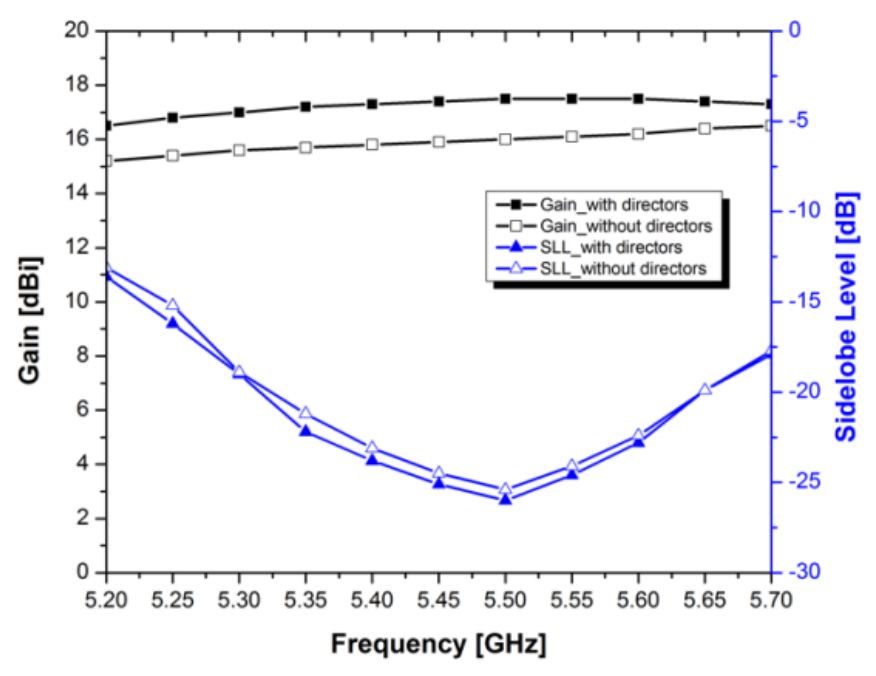

Figure 13. The gain and SLL over the operating bandwidth.

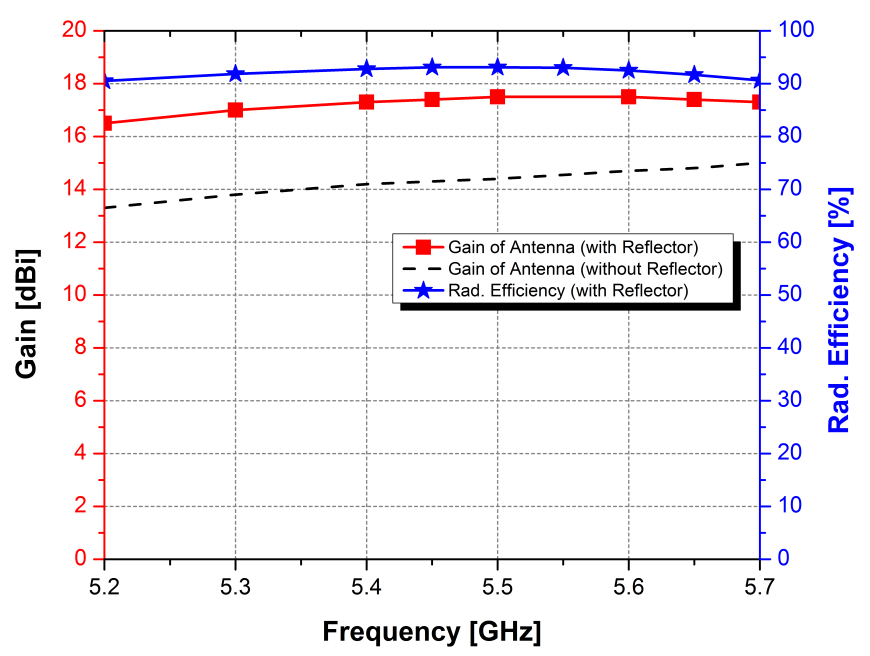

Figure 14. Comparison of gain between the array with and without reflector.

Figure 16 shows the simulated and measured return loss of the antenna. It can be seen that the operating frequency of the antenna is $5.5 \mathrm{GHz}$ with the bandwidth (at $S_{11}=-10 \mathrm{~dB}$ ) of $212 \mathrm{MHz}$.

The simulated and measured radiation patterns in $\mathrm{E}$ and $\mathrm{H}$ plane are also compared and presented in Figure 17.

It is clear that simulated data have agreed well with measured ones. Indeed, the gain in simulation and measurement are $17.5 \mathrm{dBi}$ and $17.1 \mathrm{dBi}$, respectively. 


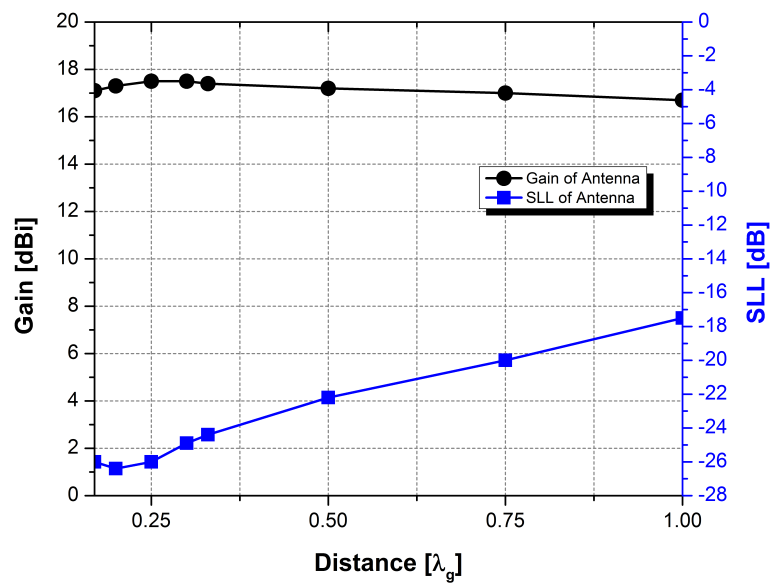

Figure 15. The variation of gain and SLL with reflector's distance to the array.

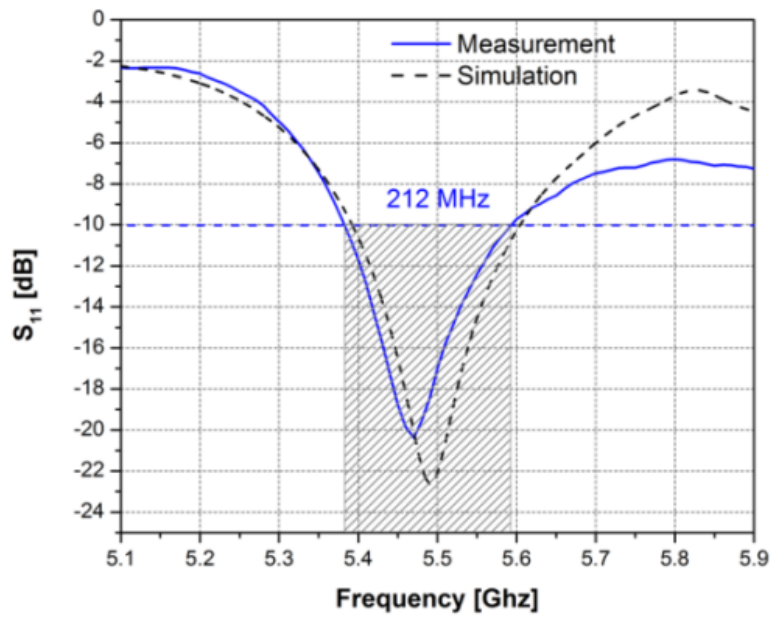

Figure 16. The measured return loss of the proposed array antenna.

Moreover, the low SLL in measurement has been also achieved with about $-25.62 \mathrm{dBi}$ compared to $-26 \mathrm{~dB}$ from the simulation. The cross polarization in both simulation and measurement has also been given and they are both lower than $-20 \mathrm{~dB}$.

The results in this work have also been collated with the other works from the literature as shown in Table VI.

It can be seen that with the same number of elements, the proposed array has the gain of $17.5 \mathrm{dBi}$, which is higher than that of [4] with just $14.5 \mathrm{dBi}$ and [6] with about $15.7 \mathrm{dBi}$. Moreover, the SLL in this work is $0.7 \mathrm{~dB}$ lower than that in [4] but $1.7 \mathrm{~dB}$ higher than that in [6]. In comparison with the work in [4], our proposed array has better results in terms of both gain and SLL. As a result of using the low complexity linear fed network, the proposed array can radiate with higher efficiency in comparison with the other works.

\section{Conclusions}

In this paper, a novel high gain and low sidelobe level linear microstrip antenna array for outdoor WLAN applications has been proposed. The design procedure

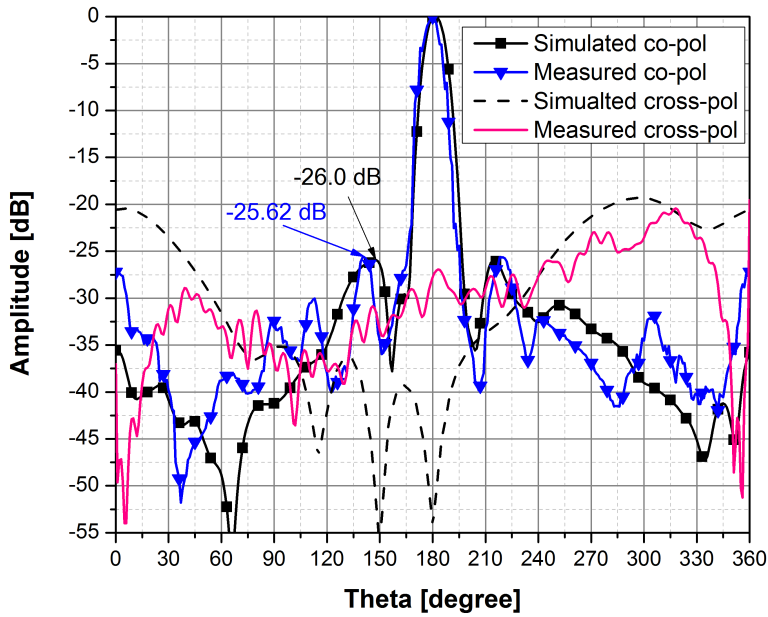

(a) H-plane.

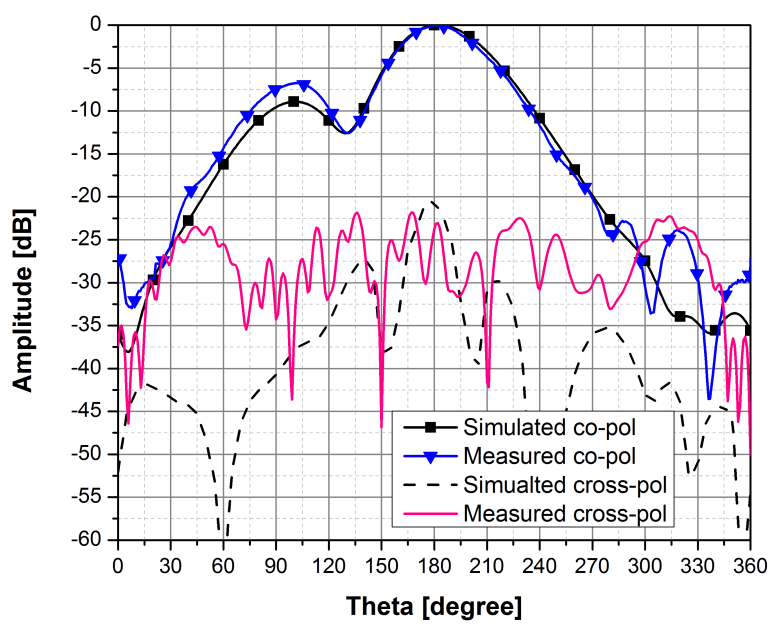

(b) E-plane.

Figure 17. Structure of the proposed array.

Table VI

The COMparison BetweEn ReFERENCES AND This Work

\begin{tabular}{|l|c|c|c|c|}
\hline References & {$[4]$} & {$[6]$} & {$[7]$} & this work \\
\hline $\begin{array}{c}\text { Substrate } \\
\text { (thickness, } \\
\text { dielectric } \\
\text { constant) }\end{array}$ & $\begin{array}{c}\text { Roger } \\
\text { RO4350 } \\
(0.508 \mathrm{~mm}, \\
3.66)\end{array}$ & $\begin{array}{c}\text { Roger } \\
\text { RT/ } \\
\text { Duroid } \\
5880 \mathrm{tm} \\
(0.2 \mathrm{~mm}, \\
2.2)\end{array}$ & $\begin{array}{c}\text { Roger } \\
\text { RT/ } \\
\text { Duroid } \\
5880 \mathrm{tm} \\
(0.508 \mathrm{~mm}, \\
2.2)\end{array}$ & $\begin{array}{c}\text { Roger } \\
\text { RT/ } \\
\text { Duroid } \\
5870 \mathrm{tm} \\
(1.575 \mathrm{~mm}, \\
2.33)\end{array}$ \\
\hline Element No. & $10 \times 1$ & $10 \times 1$ & $8 \times 1$ & $10 \times 1$ \\
\hline Size $(\lambda)$ & $6.01 \times 0.37$ & na & na & $7.7 \times 1.83 \times 0.19$ \\
\hline Freq. (GHz) & 9.0 & 60 & 7.54 & 5.5 \\
\hline bandwidth (\%) & 1.3 & 36.1 & 31.9 & 3.9 \\
\hline Gain (dBi) & 14.5 & 15.7 & 15.7 & 17.5 \\
\hline SLL (dB) & -25.3 & -27.7 & -23.1 & -26.0 \\
\hline Cross-po (dB) & -25.0 & na & -30.0 & -20.0 \\
\hline $\begin{array}{l}\text { Radiation } \\
\text { efficiency (\%) }\end{array}$ & 71 & 92 & na & 93.1 \\
\hline na: not available & & \\
\hline
\end{tabular}

from the single element to full array construction has been presented in details. In order to get low SLLs, Chebyshev weighting method has been deployed in the feeding network of the antenna. The Chebyshev excitation coefficients have been obtained in the feed by using the shunt stubs added at the feed line of each 
element. The simulation results show that the array can work at $5.5 \mathrm{GHz}$ with the bandwidth of $212 \mathrm{MHz}$ and has a high gain of $17.5 \mathrm{dBi}$ with the low SLL of about $-26 \mathrm{~dB}$. A prototype has also been fabricated and measured to validate the simulation results. It is clear from the comparison that simulated and measured data agree well with each other.

\section{ACKNOWLEDGMENTS}

This work has been partly supported by Vietnam National University, Hanoi (VNU), under Project No. QG. 16.27 .

\section{REFERENCES}

[1] J. Xiao, G. Dong, and M. Zhu, “A novel aperture coupled microstrip antenna array with low cross-polarization, low sidelobe and backlobe," in Proceedings of the $4^{\text {th }}$ International Conference on Microwave and Millimeter Wave Technology. IEEE, 2004, pp. 223-226.

[2] Y. P. Saputra, F. Oktafiani, Y. Wahyu, and A. Munir, "Side lobe suppression for $x$-band array antenna using dolphchebyshev power distribution," in Proceedings of the 22nd Asia-Pacific Conference on Communications (APCC). IEEE, 2016, pp. 86-89.

[3] C. Niu, J. She, and Z. Feng, "Design and simulation of linear series-fed low-sidelobe microstrip antenna array," in Proceedings of the Asia-Pacific Microwave Conference (APMC 2007). IEEE, 2007, pp. 1-4.

[4] J. Yin, Q. Wu, C. Yu, H. Wang, and W. Hong, "Lowsidelobe-level series-fed microstrip antenna array of unequal interelement spacing," IEEE Antennas and Wireless Propagation Letters, vol. 16, pp. 1695-1698, 2017.

[5] M. R. Nikkhah, J. Rashed-Mohassel, and A. A. Kishk, "A low sidelobe and wideband series-fed dielectric resonator antenna array," in Proceedings of the 21st Iranian Conference on Electrical Engineering (ICEE). IEEE, 2013, pp. 1-3.

[6] W. Shen, J. Lin, and K. Yang, "Design of a V-band low sidelobe and wideband linear DRA array," in Proceedings of the Progress in Electromagnetic Research Symposium (PIERS). IEEE, 2016, pp. 477-480.

[7] J. Lin, W. Shen, and K. Yang, "A low-sidelobe and wideband series-fed linear dielectric resonator antenna array," IEEE Antennas and Wireless Propagation Letters, vol. 16, pp. 513-516, 2017.

[8] R. Bayderkhani and H. R. Hassani, "Wideband and low sidelobe linear series fed Yagi-like antenna array," Progress In Electromagnetics Research, vol. 17, pp. 153-167, 2009.

[9] - "Low sidelobe wideband series fed double dipole microstrip antenna array," IEICE Electronics Express, vol. 6, no. 20, pp. 1462-1468, 2009.

[10] N. Bayat, H. Hassani, and S. M. A. Nezhad, "Sidelobe level reduction in microstrip patch antenna array," in Proceedings of the Loughborough Antennas and Propagation Conference (LAPC). IEEE, 2011, pp. 1-4.

[11] R. Bayderkhani and H. R. Hassani, "Wideband and low sidelobe slot antenna fed by series-fed printed array," IEEE Transactions on Antennas and Propagation, vol. 58, no. 12 , pp. 3898-3904, 2010.

[12] T. T. Toan, N. M. Tran, and T. V. B. Giang, "A new approach to design and optimize double-sided printed dipole antennas," in Proceedings of the 2016 National Conference on Electronics, Communications, and Information Technology, 2016, pp. 2/21-2/24.

[13] T. L. Wu, Microware filter design. Department of Electrical Engineering National Taiwan University, 2014.
[14] T. T. Toan, N. M. Tran, and T. V. B. Giang, "A low sidelobe fan-beam series fed linear antenna array for IEEE 802.11 ac outdoor applications," in Proceedings of the International Conference on Advanced Technologies for Communications (ATC). IEEE, 2017, pp. 161-165.

[15] _ - "Designing a feeding network of linear antenna arrays using Chebyshev distribution weights for a sidelobe level of $-27 \mathrm{~dB}, "$ in Proceedings of the Vietnam Japan Microwave 2017 Conference, 2017, pp. 45-49.

[16] Y. Huang and K. Boyle, Antennas: from theory to practice. John Wiley \& Sons, 2008.

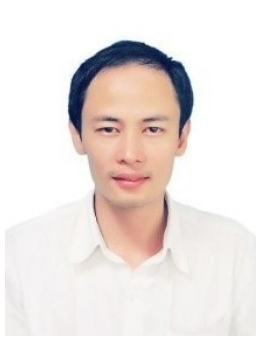

Tang The Toan is the Dean of the Faculty of Communication Technology, University of Hai Duong, and also is a senior Ph.D. student at Faculty of Electronics and Telecommunications, University of Engineering and Technology, VNU. He received his Bachelor's degree in Physics from Hanoi University of Education (2004) and his Master's degree in Electronics from University of Engineering and Technology (2011).

His main research interests are electromagnetic field theory and applications, antennas, array antennas, metamaterials, and electromagnetic band gap.

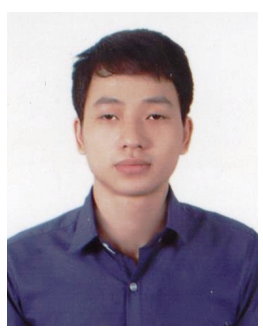

Nguyen Minh Tran received the B.S. degree and M.S. degree in Electronics and Telecommunication Technology from the University of Engineering and Technology, VNU in 2014 and 2016, respectively. He is currently pursuing his Ph.D. degree in the College of Information and Communication Engineering, Sungkyunkwan University, South Korea, since 2017.

His research interests include microstrip antennas analysis and design, coding metamaterial, RF circuit design and RF power transfer.

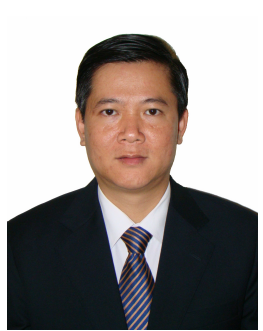

Truong Vu Bang Giang received the B.S. and M.S. degree from the VNU-University of Sciences, in 1994 and 1997, respectively, and the Dr.-Ing. (Ph.D.) degree in Electrical Engineering from the Hamburg-Harburg University of Technology, Hamburg, Germany, in collaboration with the Institute of Communications and Navigation, German Aerospcae Center, in 2006.

He is now the Deputy Director of Science and Technology Department of Vietnam National University, Hanoi and as the Secretary of the National Research Program for Sustainable Development of North-West Region of Vietnam.

He is currently the Vice President of Radio Electronics Association of Vietnam; Member of IEEE MTTs and APS. He has served as the Steering Committee (Co-Chair), Organizing Committee (Chair and Co-Chairs) or Technical Committee of ATC, REV-ECIT, VJMW, VJISAP conferences in Vietnam; Scientific and Technical Committee, International Transaction Journal of Engineering, Management, \& Applied Sciences \& Technologies (ITJEMAST)

His current research interests include Microstrip Antennas for Mobile and Handheld Devices; Analysis and Design of Conformal Antennas; Digital Beamforming and Beamsteering for Smart Antennas; Metamaterial Antennas; Design of RF Devices and Systems. 